\title{
Multilevel Social Spaces: The Network Dynamics of Organizational Fields ${ }^{1}$
}

\author{
James Hollway \\ The Graduate Institute \\ Chemin Eugène-Rigot 2, 1211 Genève (Switzerland) \\ Email: james.hollway@graduateinstitute.ch
}

Alessandro Lomi

University of Italian Switzerland

Via Buffi 13, 6900 Lugano (Switzerland)

Email: alessandro.lomi@usi.ch

Francesca Pallotti*

University of Greenwich

Old Royal Naval College, Park Row, London SE10 9LS (UK)

Email: f.pallotti@gre.ac.uk

\section{Christoph Stadtfeld}

ETH Zürich

Clausiusstrasse 50, 8092 Zürich (Switzerland)

Email: christoph.stadtfeld@ethz.ch

\footnotetext{
${ }^{1}$ We acknowledge financial support from the Swiss National Science Foundation (Grant number 146763). The names of the authors are reported in alphabetical order. Each contributed equally to the paper.

• Corresponding Author: Francesca Pallotti (f.pallotti@gre.ac.uk)
} 


\title{
Multilevel Social Spaces and the Network Dynamics of Organizational Fields
}

\begin{abstract}
We consider the dynamics of organizational fields as driven by multilevel micro-mechanisms connecting internal and external organizational decisions. The former type of change involves organizational decisions to adjust portfolios of internal activities. The latter kind of change involves organizational decisions to modify portfolios of ties with network partners. The aggregate result of these change processes is a system of interconnected positions that organizations occupy in a multilayered social space. We explain how organizations move through the multilevel space generated by their interconnected decisions by specifying newly derived stochastic actor-oriented models for the analysis of network change across multiple levels. We use data that we have collected on organizational and network change in a field of health care organizations to examine the role of multilevel mechanisms in producing and reproducing the complex structure of collaborative relations commonly observed in empirical studies of interorganizational networks.
\end{abstract}

Keywords: Multilevel models; Mixed triad census; Collaboration networks; Affiliation networks; Organizational fields; Multilayer networks; Interorganizational networks; Organizational change; Social spaces 


\section{Introduction}

Contemporary research on social networks increasingly recognizes that generative social mechanisms rarely operate independently of the positions actors occupy in a more general social space emerging from the "interdependence of relationships among different types of social entities, such as persons, groups, sociocultural resources and places" (Pattison \& Robins, 2004: 11). The concept of social space has frequently been invoked to describe how structural and physical features of networks jointly impinge on actors' decisions and behaviour (Daraganova et al., 2012; Mische \& White, 1998; Pattison \& Robins, 2002). Padgett and Ansell (1993), for example, present a case in which social identities and individual strategies can only be understood with reference to the positions that actors occupy in a multilevel social space. While the notion of social space is not new (Bourdieu, 1985; 1989), two recent parallel developments in the study of social networks help to clarify its operational definition.

First, recent advances in the analysis of multilayer networks provide the basic analytical concepts for understanding social spaces as constructed by multiple, intersecting networks (Kivela et al., 2014: Table 1). Examples of contexts for interactions that have been used to identify aspects of social spaces include: activities in which social actors are involved (Lomi \& Stadtfeld, 2014); events they attend (Ingram \& Morris, 2007); projects in which they participate (Mische \& Pattison, 2000); preferences they express (Snijders et al., 2013); institutional categories used to classify them (Mohr, 1994); and resources or information they control (Carley, 1991). In all these cases, social space can be extended into multilayer or multilevel combinations of associative (one-mode) networks connecting social actors, and affiliative (two-mode) networks linking social actors to various social foci (Wasserman \& Iacobucci 1991; see also Iacobucci \& Wasserman, 1990). This intuition has stimulated considerable interest recently in the analysis of what scholars of different 
disciplines variably call multilayer, multiplex, and multilevel networks (Gluckman, 1955; Verbrugge, 1979; Wasserman \& Faust, 1994; Lazega et al., 2008; Lomi et al., 2016; Snijders, 2016; Wang et al., 2013; Kivelä et al., 2014).

Second, the advent of statistical models for representing the dynamics of network structures provides us with the opportunity to incorporate a temporal aspect into the notion of social space. To date, most of the work on social network dynamics has relied on stochastic actor-oriented models or SAOMs (Snijders, 2001; 2010). More recently, extensions of SAOMs have been derived to study how one network structure coevolves with actors' behaviour (Steglich et al., 2010) and with other networks, including two-mode networks (Snijders et al., 2013). Models for coevolving one-mode and two-mode networks provide one potential opportunity to develop an integrative view of these parallel developments in the analysis of multilevel and multilayer networks (see Snijders, 2016; e.g. Milewicz et al., forthcoming).

Against this general background, in this paper, we seek to advance an updated concept of social space that integrates the multilayer and dynamic statistical network methods currently at the disposal of social network researchers. We demonstrate the analytical value of the new concept of social space that we propose with the help of an illustrative analysis of an organizational field - a community of interacting organizations that "in the aggregate constitute a recognized area of institutional life" (DiMaggio \& Powell, 1983: 148). Organizational fields are well suited to our current analytical purpose because external and internal organizational changes (or "decisions") congeal into a multilevel system of action that shapes the space of possibilities for other participants in the field (DiMaggio, 1986; Padgett \& Powell, 2012). In other words, decisions to change internal structures by modifying portfolios of organizational activities and decisions to change external relations by modifying portfolios of relations with partners create interdependencies across 
multiple levels of action (Stadtfeld et al., 2016). Through these internal and external decisions, organizations seek certain positions in their social space while simultaneously modifying that space. We consider these decisions as giving rise to a multilevel system of action because the resources that an organization can mobilize include internal resources that it can control directly through investment in production activities, and extramural resources controlled by partners that the organization may access indirectly through investments in relations with network partners (Cohen \& Levinthal, 1994). These (internal and external) investment decisions produce change in organizational and network structures, respectively.

To explore the analytical value of this view, we use longitudinal data that we have collected on a field of hospital organizations rendering health care services to a local population of approximately 6 million people. For our purposes, the primary external decision of consequence concerns the selection of collaboration partners. More specifically, we focus on patient transfers as one of the most frequently studied collaborative relationship observed among hospitals (Iwashyna, 2012; Iwashyna et al., 2009; Lomi \& Pallotti, 2012; Veinot et al., 2012). Over time, hospitals may decide to collaborate by sharing clinical cases and knowledge with other hospitals. This collaboration involves transferring patients to established partners and/or to new partners (Lomi et al., 2014b). Individual partner selection decisions aggregate into a decentralized network of collaborative and joint problem-solving relations among the members of the organizational field, and subsequent decisions depend also on the network structure in which potential collaboration ties are embedded (Veinot et al., 2012).

Partner selection decisions also depend on considerations of compatibility between potential partners. Compatibility is evaluated with respect to the resources, knowledge, and activities that potential partners may be willing to contribute to their potential collaboration (Mitsuhashi \& Greve, 
2009). For this reason, the primary internal decision of consequence is what portfolio of activities to maintain. Organizations choose to invest in specific activities and in so doing accumulate specific knowledge and resources (Cohen \& Levinthal, 1994). The sum of these internal portfolio decisions for each organization represents the internal organizational structure that is observable at any one time. Where organizational activities overlap across organizations they may be interpreted as contexts for interaction or foci (Feld, 1981) because they provide the basis for assessing complementarities that in turn shape both social selection and social influence processes (Cohen \& Levinthal, 1990; Greve, 2005; Haunschild \& Beckman, 1998). In the empirical part of the paper, we represent the internal structure of the hospitals in terms of the set of clinical specialties (or activities) they include. Over time, organizations may decide to change their internal portfolio of activities by investing in new or abandoning old activities. These decisions depend on the internal resources they have available, but also on how they can fulfill their functions and remain competitive through establishing network ties with partner organizations (Cohen \& Levinthal, 1994). In organizational fields, the link between internal and external choices is produced by the mutual awareness implied by field membership (DiMaggio \& Powell, 1983).

We argue in this paper that the interlocking of these sets of internal and external change decisions constitutes a social space through which organizations move over time. Organizations change their positions in this multilevel social space by changing their collaborative ties with other organizations and by changing their portfolio of activities. Thus, the social space represented in the models we propose changes as each organization tries to solve their individual "make or cooperate" problem (Kogut et al., 1992), and in so doing changes their position over time.

The following section elaborates this notion of social space, updating it with respect to three key developments in the statistical modeling of social networks: multilevel, dynamics, and structural. 
We then introduce our empirical setting with reference to how networks of organizations' internal and external decisions with respect to their competition and collaboration interlock, forming an emergent, multilevel social space through time. The fourth section introduces the data in more detail and proposes a number of mechanisms operating in one or both networks that we would expect to recreate the multilevel topology of the social space over time. In section 5, we present our results, paying particular attention to model sufficiency with regards to the goodness of fit statistics and plots. We conclude by arguing that network researchers' choices of goodness of fit statistics should reflect a consideration about the dimensions of social space of most interest to the nodes involved.

\section{Updating the Concept of Social Space}

According to Abbott (1997: 1152): “One cannot understand social life without understanding the arrangements of social actors in particular social times and places." In the notion of "social space" that we elaborate below, we connect Abbott's notions of "social place," "social time" and social "arrangements", to the notions of "multilevel structure," "network dynamics, " and "local structure" - respectively. Our attempt to provide a new operational definition of social space originates from the observation that "no social fact makes any sense abstracted from its context in social (and often geographical) space and social time" (Abbott, 1997: 1152).

Pattison \& Robins (2004: 14) argue that understanding social space requires "Attention to many types of entities, including those referring to geography, social settings, affiliations, social relationships, and the distribution of cultural resources." This statement provides the basis for the definition of social space that we seek to articulate and forward in this paper. However, by developing the concept of social space, we differ from available conceptualizations (Pattison \& 
Robins, 2002; 2004) in three key ways as we consider social spaces as (i) multilevel, (ii) dynamic, and (iii) structural.

\subsection{Social Space as Multilevel}

First, Pattison and Robins (2002; 2004) only define social space in relation to single networks, whether one-mode as in the case of the Bank Wiring Room or two-mode as in the case of the Southern Women dataset. In line with this special issue on multilayer networks and considerable recent work in social networks, we see here an opportunity to integrate the potential dimensions of social space Pattison and Robins introduce above into a more expansive definition.

Indeed, the extension to multilayer or multilevel networks is relatively unproblematic. Just as the triad census captures local neighborhood patterns surrounding individual nodes in a single network (Holland \& Leinhardt, 1970; see Pattison \& Robins, 2002: 303-304), a multilevel triad census can be defined for the local neighborhoods that span networks. Snijders and Stokman propose a census of triads that also span subgroups, which could include other node sets (Snijders \& Stokman, 1987). The multilevel triad census presented here is in line with the idea of triad census for one-mode networks (Davis \& Leinhardt (1972) and two-mode networks (Brunson, 2015), but extends to counting structures across two types of networks simultaneously. Figure 1 reports the possible motifs in the multilevel triad census (MLTC).

Insert figure 1 about here

In Figure 1, circles represent organizations (in our empirical example hospitals) and squares represent the internal activities available to them (clinical specialties). Directed edges connecting circles record the presence and direction of collaborative ties between organizations. Undirected 
edges affiliate organizational nodes to activities. The two-digit labeling convention we follow is straightforward. The first digit tells the number of undirected edges in the motif (i.e., the number of undirected edges affiliating the circles at the base with the square at the top). The second digit tells the number of directed edges in the motif (i.e., the edges between the two circles at the base of the triangle). Thus, for example, the triadic multilevel motif 22 involves the presence of reciprocated ties between organizations (hospitals) affiliated to the same activity (clinical specialty). Multilevel motif 20 indicates the absence of any relation between organizations affiliated with the same activity. In case one tie of each type is present (coded as 11), the two possible multilevel motifs are distinguished by appending letters $D$ (down) and U (up) that refer to the direction of the tie connecting the circles.

Triadic multilevel motifs 22, 21, 20,12, $11 \mathrm{D}$ and $11 \mathrm{U}$ are of particular interest because they reveal the presence of elementary multilevel configurations - and hence multilevel dependencies - in the data. Multilevel motif 22 captures a major form of multilevel closure - namely, the preferential tendency of organizations sharing the same activity to entertain mutual collaborative exchange relations. Multilevel motif 21 captures the preferential tendency of organizations to connect with partners affiliated to the same activity. Both motifs 22 and 21 may be conceptually associated with the expectation that organizations sharing activities (and hence knowledge base) will be better able extract value from potential partnership (Cohen \& Levinthal, 1990). For this reason, according to absorptive capacity arguments, organizations with similar internal activities are expected to be more likely to select one another as partner. Motif 20 captures the opposite tendency: the lack of direct relations between organizations sharing activities. Similarly, motif 12 is of interest because it may be considered as a possible antecedent of 22; organizations might learn (useful combinations of) activities from collaborative partners. Finally, interest in motifs $11 \mathrm{U}$ and 22D may be justified 
because it reveals the tendency of organizations to prefer complementary - rather than similar partners. In this sense, a social space can be extended to a special kind of heterogeneous information, or multilevel, network containing multiple kinds of nodes linked by multiple kinds of edges (Kivela, 2014; Lomi et al., 2016). The advantage of doing so is that we capture more of the social context in which actors, in our case hospital organizations, operate.

\subsection{Social Space as Dynamic}

Second, while Pattison \& Robins explicated an approach for mapping social space that relied on cross-sectional ERGMs, here we wish to extend this view by incorporating explicitly temporal variation. In part, the extension we propose is driven by the possibilities afforded by newly derived stochastic actor oriented models (SAOMs) for the coevolution of one- and two-mode networks (Snijders et al., 2013). However, the motivation also comes from our objective to conceptualize social space as something that actors move through over time. Indeed, Pattison \& Robins themselves endorse Emirbayer's view that the "social world consists primarily in 'dynamic, unfolding relations' rather than in substances and 'static "things"'" (Pattison \& Robins, 2004: 15 citing Emirbayer, 1997: 781, emphasis in original). In our conception, actors have trajectories through a social space constituted by relationships between and among them that aggregate analytically to network representations. This means that actors change their social space as they move through it. This malleability of social space is key to theories of social construction and is perfectly integrable into this conception. Social space is thus not an equilibrium state but a medium, or context for dynamic action. It follows, therefore, that methods investigating social space should allow for topological change.

We adopt stochastic actor-oriented models (SAOMs) for multilevel networks (Snijders et al., 2013) that allow one to test in how far competing dynamic explanations contribute to changes between 
empirically observed network panel data. Snijders et al. (2013) introduce SAOMs for multilevel networks. The core idea is that actors over time optimize their portfolio of outgoing ties on two network levels simultaneously. Network changes are modeled as discrete choices of actors about creating, dropping or maintaining network ties. These discrete choices are embedded in a continuous-time Markov processes. The utility of an actor who considers changing a network tie is controlled by two linear "evaluation functions," that are defined in equation 1 and 2 in Snijders et al. (2013):

$$
\begin{aligned}
& \mathrm{fYi}(\mathrm{x}, \mathrm{y})=\sum \mathrm{k} \beta \mathrm{Yk} \text { sYki }(\mathrm{x}, \mathrm{y}) \\
& \mathrm{fXi}(\mathrm{x}, \mathrm{y})=\sum \mathrm{k} \beta \mathrm{Xk} \text { sXki }(\mathrm{x}, \mathrm{y})
\end{aligned}
$$

The functions sYki and sXki are so-called objective functions that measure structures in the current networks $\mathrm{X}$ and $\mathrm{Y}$ (the two network levels) from an actor i's perspective and take into account additional variables. These functions represent an actor's perception of the network. In our specific empirical case, for example, an effect might measure how many ties a hospital maintains in either of the two networks, how many collaborative ties it has with hospitals nearby, or how many specialties it shares with hospitals with which it is collaborating. Each of these effects is evaluated by a parameter $\beta \mathrm{k}$ that may be estimated from suitable data. Positive parameters indicate that ties are related to increases in the underlying statistic (e.g., having more collaborative partners). The effect specifications of the models in this paper are discussed in the following section. Additional mathematical details are provided in Snijders et al. (2013) and Stadtfeld et al. (2016).

Note that SAOM parameters are estimated here using the method of moments. The key challenge in the estimation procedure is that dynamic networks of relational states are commonly measured as panel data but SAOMs are continuous-time models of network change. Therefore, the estimation 
routines for SAOMs make use of simulations to generate potential change processes. The approach we use for the estimation in this paper uses the method of moments and tries to find parameters so that repeated simulations of the change process between all subsequent waves return networks that are similar (in terms of method of moments target statistics) to the observed ones. Details about the estimation routine are reported in Ripley et al. (2015).

Our point here is not confined to SAOMs specifically. The important move, we argue, is to update the concept of social space such that it captures how it changes over time as social actors move through it by repositioning themselves within it. Social space is not static, but malleable by both the intentional and unintentional actions of actors within it. Such dynamics could be captured just as well by alternative models, however we find the multilevel SAOM particularly useful for illustrating this conceptual development.

\subsection{Social Space as Structural}

Third, while we agree with Pattison \& Robins that the elements of social space are relational and should be viewed as stochastic (Pattison \& Robins, 2004: 16), our view differs in how it conceives of the relationship between model and social space. Take two examples offered in the most recent formulation of social space (Pattison \& Robins, 2004): the Bank Wiring Room and Southern Women datasets. They characterize the former in terms of "a connected structure in which the variation in nodal degree is moderate but not high and in which local relational clustering prevails" (p. 21). For the latter they argue that while there is evidence for "local preferential attending patterns" there is also "local clustering in the relational space" (p. 24). In each case, social space is clearly defined relationally, but also in terms of generative relational social mechanisms like preferential attachment and clustering. We argue that social space and the mechanisms, relational or otherwise, that generate and transform social space should be distinguished. In other words, for 
Pattison and Robins, the model defines the social space; for us, the model's purpose is to adequately replicate or reconstruct the social space.

Therefore, we propose that social space be associated with summary distributions of local structures, rather than with the individual mechanisms that may generate these distributions. In order to assess whether an estimated model fits the data well, we use the goodness-of-fit (GOF) test in the RSiena software (Ripley et al., 2015) as proposed by Lospinoso (2012). It allows us to investigate whether data features that were not explicitly modeled as method of moments target statistics are nonetheless well represented in the networks simulated during the method of moments estimation procedure. Separating the concept of social space from relational mechanisms expands it significantly, as it can capture how social space represents the concatenation of multiple mechanisms, relational and otherwise, as constituted in multiple, multilevel networks. It also empowers GOF tests, imbuing them with meaning and purpose beyond being "auxiliary" checks.

Of particular interest in this paper is whether the multilevel triad census (MLTC) that we introduced in figure 1 is well captured by simulations of network evolutions generated using the estimated parameters. In order to assess this, a new GOF function ("MixedTriadCensus") was added to the RSiena software. This mixed triad census helps us gauge how well we capture multilevel social space over time in a given model in terms of summary distributions of local structures. In the results section of the paper we will evaluate whether dynamic models of different levels of complexity allow us to simulate networks with a MLTC that is similar to the empirically observed MLTC. A model with an excellent fit regarding MLTC will simulate networks so that the empirically observed MLTC will be close to the center of the simulation distributions for each of the motifs in figure 1. This would mean the model better represents the multilevel social space, through time, of an organizational field of hospitals. This is not just a post-hoc diagnostic test, we argue. Such 
goodness-of-fit plots help us gauge how well the social space in which actors' interdependent decisions are embedded is captured by a model. It is thus more meaningful than post-hoc diagnostic test. Thus also the researcher's choice of which structural dimensions of social space to recreate becomes a more meaningful, even theoretic one. Here we have chosen the multilevel triad census introduced above as the most meaningful dimension of social space for our hospital organizations, as it is how collaboration and specialty networks interlock that is crucial for understanding patterns of collaboration and competition as hospitals decide whether to "make or cooperate". The next section relates these three developments to the concept of social space - multilevel, dynamic, and structural - to our specific empirical example of an organizational field of hospitals.

\section{The Multilevel Social Space of an Organizational Field of Hospitals}

The empirical case we develop in this paper is the dynamic, multilevel social space of a field of hospital organizations. More specifically, the field consists of 110 hospitals providing health care services in Lazio, the second largest region in Italy with a population of approximately 6 million inhabitants and containing the largest Italian city, Rome. The regional health system in Lazio is part of the Italian National Healthcare System (Servizio Sanitario Nazionale, SSN). The SSN provides universal health assistance to all citizens and residents free of charge at the point of service. The SSN has a federal structure organized at the regional level. Within each region such as Lazio, responsibility for the organization and delivery of services rests on geographically and population-defined institutions called Local Health Units (LHUs). LHUs coordinate the services rendered by public as well as private accredited hospitals located in their referent geographical area. These are not hard boundaries though; patients are free to seek health care from any health care provider located within or outside their LHU of residence. The regional health system in Lazio is organized around 12 LHUs, 8 of which are concentrated in the capital city (Rome) and 4 LHUs 
covering the area of the other four provinces of the Region (Rieti, Latina, Viterbo and Frosinone). Across the Region, each LHU is responsible for the provision of services to approximately 500,000 residents. LHUs in Lazio have been designed to be relatively self-contained, i.e., to be able to provide a wide range of care services to their target patient population. These 110 hospitals are the organizational actors whose social space we are interested in representing and understanding.

These hospitals are part of an organizational field that encourages coordination and collaboration as well as competition to best serve the interests of the patients and thereby fulfil their primary organizational function (Lomi \& Pallotti, 2012). Hospitals take two main types of decisions that affect the social space within which hospitals operate. The first is the decision to transfer a patient and the related choice of a destination hospital for the patient. This gives rise to a one-mode network of inter-hospital collaborative relations. The second is the decision to change the internal structure of organizational activities, i.e. to add or abandon clinical specialties. This gives rise to a two-mode network of hospitals by clinical specialties. The social space of the Lazio regional health system is thus multilevel because it is the result of two interlocking networks based on a set of external and internal decisions to capture the social space of these organizations.

The first of these two networks consists of patient transfer relations between the hospitals. An interhospital patient transfer occurs when a patient discharged from one ("sender") hospital is admitted to another ("receiver") hospital in the same calendar day. Inter-hospital patient transfers are an essential component of health care systems (Sethi \& Subramanian, 2014). Proliferation of specialties and regionalization of specialized care have contributed to an increase in inter-hospital patient transfers (Wagner et al., 2013). Over the last decades the health care literature has devoted increasing attention to these transfers, not only because there are economic costs and clinical risks 
associated with them, but also because they often play a critical role in ensuring the best possible medical care to patients (Hains et al., 2011; Lomi et al., 2014b).

Patient transfer is considered an important form of inter-hospital collaboration (Lee et al., 2011; Iwashyna \& Courey, 2011; Iwashyna et al., 2009; Lomi \& Pallotti, 2012; Veinot et al., 2012; Pallotti et al., 2015). Recent work examining patient transfers has shown that mutual coordination, communication and sharing of information between partners are key to the quality and safety of these transfers (Iwashyna et al., 2009; Iwashyna, 2012; Lomi et al., 2014b; Hains et al., 2011). The underlying goal of patient transfers is to provide continuity of care to patients whose pathologies require collaboration between hospitals. Partner selection decisions are based, at least in part, by considerations of quality, infrastructure, proximity, and familiarity (Kitts et al., 2013; Lomi et al., 2014b; Iwashyna \& Courey, 2011). Once the decision is made, completing a transfer requires not only a physical and technical infrastructure making the transfer operationally possible (Iwashyna, 2012), but also a relational infrastructure based on a complex coordination and information sharing process between partner hospitals (Gittell, 2002; Bosk et al., 2011). The correct functioning of these infrastructures are essential for avoiding delays and maintaining continuity of medical care (Hains, 2011; Robinson et al, 2009).

In our analysis, we concentrate specifically on the transfer of hospitalized patients or 'non-urgent transfers' that are "considered routine and (do) not involve an immediate threat to life or limb, or care that is time-sensitive" (Robinson et al., 2009: 57). In most cases, transfers are needed when diagnostic and therapeutic facilities required for a patient are not available in the referring hospital, or when the hospital has patients with medical or clinical needs that may be met more effectively and efficiently in another hospital. In the case of non-urgent transfers, the choice of the destination hospital involves an explicit partner selection decision: a transferring hospital is free to select its 
partner from a number of potential receiving hospitals. Therefore, building on fieldwork and extant research, we treat the presence of non-urgent patient transfer relations as the result of decisions to collaborate with external partners.

The second of these two networks consists of relations affiliating hospitals to clinical activities or specialties they maintain. These relations may be viewed as the result of internal choices and are integrated into internal organizational structures. Of course, the same specialties may be maintained by multiple hospitals. In this case, hospitals choose which of a recognized list of clinical specialties, for example cardiology, oncology, pediatrics, or geriatrics, to maintain over time. Hospitals' choices affect their social space. Hospitals that overlap in their choice of specialties may find themselves in competition for patients or other resources and those that do not overlap significantly in specialties may be complementary to one another.

The decision to change the internal structure of clinical activities is particularly complex for public hospitals, which enjoy lower levels of managerial autonomy than private hospitals. Investment and divestment decisions of public hospitals are also subjected to stronger institutional constraints imposed by public administration on the availability of financial, human and political resources. Private hospitals, on the other hand, are typically subjected to the competitive constraints imposed by the market for health care services. As such, it is easier to assimilate the decision to add or abandon clinical activities to a diversification or refocusing decision faced by more conventional business organizations.

In this paper, we adopt a view of hospitals as composite agents that choose their (external) collaborative partners and the (internal) activities they specialize in interdependently, giving rise to a single, multilevel social space within which the two networks coevolve. As we have defined it, a social space (rather than two independent networks) exists to the extent that these apparently 
independent sets decisions generate - and at the same time are affected by - multilevel dependencies that connect processes of external and internal organizational choices. Both change and constitute a context for the other and even the mechanisms driving rather small changes in one are required to understand the dynamics of the other.

For example, the decision to refer a patient to a specific partner hospital is influenced by the types of services that each of the two hospitals provide. Indeed, "Similarity of internal or external characteristics affects judgments of relevance that an origin organization has to a focal organization, and hence influences the rate of interorganizational learning" (Greve, 2005: 1032). Accordingly, similarity in portfolios of internal organizational activities (i.e., overlapping affiliation to the same organizational activities) affects partner selection decisions and hence the dynamics of interorganizational networks.

For the same reason, a hospital that receives patient transfers from many hospitals collects resources that enable organizational growth or at least maintenance of key clinical specialties and a hospital that finds itself transferring many patients may seek to stem this flow by expanding to specialties it finds itself lacking. As such, changes in the internal composition of organizational activities are particularly important as they imply changes in the levels of complementarity and similarity between partner hospitals.

The component elements of the social space that we have identified change over time as a consequence of organizational decisions to adjust portfolios of internal activities in response to change in the availability of actual and potential resources, and to change in portfolios of network ties in response to change in the availability of actual and potential partners. This application of our multilevel, dynamic definition of social space to our case of hospital organizations can serve 
as context to gauge how useful or adequate an account it would offer of the mechanisms underlying organizations' actions within and movement through this space

\section{Data, Variables and Mechanisms}

Our analysis relies primarily on information contained in public records maintained in the Regional Hospital Information System database (SIO), which is managed by the Public Health Agency of Lazio (Agenzia di Sanita' Pubblica - ASP). This is a large administrative dataset, containing a wide range of information on patients, hospitals, and outcomes.

The dataset that we analyze is the result of a five-wave network panel design. The overall observation period is 2006-2010. We collected information on dyadic relations defined in terms of patient transfers among all the 110 public and private accredited hospitals in the Lazio region. During the period of observation, 82,440 patients were transferred between hospitals in our sample. Using these data we constructed 5 patient transfer matrices containing in rows (columns) the sender (receiver) hospitals, and in the intersection cells the number of patients transferred from row to column hospitals for each of the five observational years. The 5 matrices are asymmetric, since for any hospital in the sample the number of patients sent typically differs from the number of patients received. Because our focus is on processes of change in collaborative network ties, rather than change in their intensity, we derived five binary matrices by noting where matrix cells included at least one patient transferred between two hospitals within that year. This simple dichotomization seems justified in this case on substantive grounds. The data concerns the transfer of elective, rather than emergency, patients. This implies that transfers are arranged between partner hospitals in advance, and that the sender hospital can choose the receiver hospital with relatively few constraints. In these circumstances even the transfer of one patient signals collaboration - and hence the result of a joint decision-making process supporting relational coordination among 
network partners. We note that alternative dichotomization rules (e.g., based on the mean cell value) would not produce networks differing significantly in terms of density. Table 1 reports the main descriptive statistics of the networks we analyze in the empirical part of the paper. The figures in Table suggest that the density of the networks - i.e., the actual number of relations relative to the total number of possible relations - fluctuates between 0.183 and 0.197 .

Insert Table 1 about here

Table 2 reports information on tie changes occurring in subsequent periods. The first four rows show the number of dyads for each period that experienced the creation $(0 \rightarrow 1$ transitions $)$ or expiration ( $1 \rightarrow 0$ transitions) of a collaborative relationship, as well as those that remained absent $(0 \rightarrow 0)$ or were maintained $(1 \rightarrow 1)$. Over the period of observation, 2,400 new links were established and 2,519 existing links dissolved. Stability between consecutive observations for the patient transfer network can be measured by Jaccard coefficients (Batagelj \& Bren, 1995). Jaccard coefficients range between 0, if all ties change, and 1, if all ties stay the same (Snijders et al., 2010). The stability of the patient transfer networks is realistically high as revealed by Jaccard coefficients ranging between 0.537 and 0.552 .

Insert Table 2 about here

We also used information collected on the organizational activities of each hospital in the sample to construct 5 two-mode matrices of 110 hospitals by 57 clinical specialties. In the intersection cells, 1 indicates that the row hospital contains the column specialty, and 0 otherwise. Table 3 reports the change statistics of the (two-mode) hospitals-by-specialties networks that we analyze. 
The figures in the Table suggest that, as expected, the portfolio of clinical specialties tend to be rather stable over time, though there are sufficient changes to warrant modeling this as a coevolving network.

Insert Table 3 about here

We specify a model that includes several exogenous variables to control for known factors that might affect interorganizational collaboration. First among these are variables related to an organization's size. We define hospital size in line with preceding literature (Stadtfeld et al. 2016) as a hospital's capacity as measured by the number of its staffed beds. Generally, we expect larger hospitals to send and receive more patients and be able to sustain more specialties. Of course, high capacity does not necessarily entail that a hospital is a preferred partner. Indeed, sometimes smaller hospitals treat more patients or offer better care for patients when beds become available there. To capture these exceptions, we include two further variables: throughput measures the number of patients discharged (equal to the number admitted) and occupancy measures the ratio of occupied beds to the total number of beds.

Hospital collaborations are also affected by geographical, administrative and institutional dimensions. All things equal, a hospital will clearly prefer to send patients to partner hospitals that are nearby. This ensures that a patient will get prompt care. As such, we expect distance, represented as an exogenous dyadic covariate measuring the distances in kilometers between each pair of hospitals, to be negative. Hospitals also face administrative incentives to transfer patients to hospitals that belong to the same Local Health Unit (LHU). This is because administrative and accounting practices mean that patients transferred to another LHU represent a resource loss not only for the transferring hospital but also the transferring LHU. As such, we would expect transfers 
within LHUs to be more common. Hospitals share other commonalities too. Each hospital in the SSN is classified as belonging to one of six profiles - a traditional LHU hospital (1), a hospital trust (2), university hospital (3), hospital of the National Institute for Scientific Research (4), a classified hospital (5), and a private accredited hospital (6). Since all hospitals in each of these categories share similarities in ownership, governance, and normative or organizational constraints, we would expect hospitals with the same institutional profile to be more likely to transfer patients to one another.

Finally, we would expect inter-hospital collaboration to be affected by hospitals' joint dependence on common resources (i.e., patients). The variable niche captures this dependence. It measures the extent to which two hospitals render services to patients with similar pathologies and who are located in the same area. This variable is constructed following Sohn's (2001) established measure of niche overlap developed specifically for hospitals. 2 Table 4 summarizes essential information on the organization-specific variables included in the empirical model specifications. Some of the variables are defined at the organizational level (i.e., monadic variables, such as occupancy and size) while others refer to hospital dyads (i.e., dyadic variables, such as geographic distance, niche overlap, or shared organizational profile or LHU membership).

\footnotetext{
${ }^{2}$ To measure niche overlap, we used data on patients' residence and major diagnostic categories (MDC) to create two-mode origin-destination matrices recording the number of patients that every row-hospital i (destination) receives from every column zip code K (origin), for each MDC (MDC=25). The resulting 25 two-mode origin-destination matrices were then aggregated, for each year, by using the total number of discharges for each MDC as weights. On the resulting five averaged two-mode matrices (one for each year of observation), niche overlap is hence computed as: where min(pik, pjk) indicates the overlap (or "intersection") in patient pools between hospital $\mathrm{i}$ and hospital $\mathrm{j}$ in municipality $\mathrm{k}$, and the weight wik indicates the proportion of all patients admitted to hospital i who come from municipality k. In Eq. (1) the numerator expresses the overall sum of niche overlaps between hospital $i$ and $j$ across all municipalities, while the denominator simply tells the niche width of the i-th hospital, i.e., the total number of patients admitted by hospital i across all municipalities. The (dyadic) coefficient, $\omega \mathrm{ij}$ may then be interpreted as the proportion of the patient pool of a hospital overlapped by another hospital. The term min(pik, pjk) requires that $\omega$ ij lies between 0 (no overlap) and 1 (maximum overlap).
} 
Insert Table 4 about here

The second category of factors that help explain the observed patterns of collaborative patient transfers are structural tendencies, or endogenous network effects. We outline first those relating to the collaborative network, then those relating to the specialties network, before discussing those that explain how these two networks interact. These effects may be interpreted as possible mechanisms connecting decisions to change portfolios of external network ties to partner organizations and portfolios of internal organizational activities.

First, as a collaborative network, we expect substantial dyadic reciprocity. Patient transfer implies a transfer of resources from the sending to the receiving hospital. This is the case because the budget follows the patient. As a consequence it is likely that such transfer will be more likely to happen under conditions of reciprocity. Clustering is a recurrent feature in organizational fields (Baum et al., 2003; Davis et al., 2003). To control for clustering, we include in our model effects associated to transitive closure and to cyclic closure, or generalized exchange (Lomi \& Pallotti, 2012).3 Lastly, to control for how reciprocated ties may be embedded in transitive relationships, we include an interaction between transitive closure and reciprocity, which we would expect to be negative because there is little need to reciprocate within a cycle. We thus expect transitive closure in the collaboration network, however, the direction of ties within triads is expected to be noncircular or hierarchical.

We expect that hospitals receiving transfers from many other hospitals demonstrate qualities that make them even more popular, though not necessarily more active. In other words, some hospitals

\footnotetext{
${ }^{3}$ Note that both of these effects are specified in geometrically weighted terms.
} 
are effectively 'sinks' for patients — presumably in a good way! We also include an assortativity effect, which we expect to be positive; hospitals that sustain many collaborative relationships are likely to form an active core of this network, whereas others that rarely collaborate remain on the periphery.

In terms of the specialty network, we include two structural effects. First, we expect that hospitals will choose specialties that many others choose (popularity). This may be because these specialties are constitutive of being a hospital, such as "day hospital" or "general medicine", or because they attract resources, such as "cardiology". Second, we expect that hospitals will choose the same specialties as those other hospitals with which they already share specialties (4-Cycles). In other words, we expect there to be coercive and mimetic institutional isomorphism, respectively, with respect to specialty choice (DiMaggio \& Powell, 1983).

Finally, we also define a number of cross-network effects to capture interdependencies between the collaborative and specialty networks. On the one hand, we expect hospitals' collaborative choices to depend on their specialties. We would expect hospitals holding many specialties to require fewer collaborative ties to provide adequate care for their patients (negative specialty activity) but receive more collaborative ties (positive specialty popularity). Hospitals are unlikely to collaborate with those hospitals holding the same specialties (negative specialty agreement), unless it is reciprocating an incoming tie (positive specialty agreement reciprocity). However, we would not expect to see such reciprocal behavior from hospitals that hold many specialties not shared by the hospital providing the incoming tie (negative specialty activity reciprocity). In this case, the hospital receiving the collaboration tie is exploiting its comparative advantage in providing a needed specialty. On the other hand, we expect hospitals' choice of specialties to depend on their collaborative ties. For example, we would expect that hospitals choose to maintain 
the same specialties as their collaborative partners (positive collaboration agreement), and even more so when collaboration is reciprocated (positive collaboration agreement reciprocity). We note that these effects specify and upgrade earlier notions of multiplex triads in interorganizational networks (Pina-Stranger \& Lazega, 2011; Shipilov \& Li, 2010) to dynamic multilevel triads as components of more general social spaces. Table 5 summarizes our discussion.

Insert Table 5 about here

\section{Results}

We estimated three stochastic actor-oriented models. The first, which we refer to as the "null" model, provides a baseline model in which only monadic and dyad-level covariates are included. In other words, the null model contains no endogenous structural effects. The second, which we refer to as the "level independence" model, takes into account covariates and endogenous network structures on both network levels (one-mode network of interorganizational collaboration and twomode network of hospital organizations' affiliations to clinical activities). However, it does not incorporate any effects that couple the dynamics of the two levels. The third is the "full" model in which cross-level network effects are included. It employs the multiplex coevolution model proposed by Snijders et al. (2013) in a model specification similar to that presented in Stadtfeld et al. (2016). Each estimation was run until estimates converged with t-ratios (including for linear combinations of parameters) all below 0.15 . Table 6 below presents the parameter estimates and standard errors for each effect. In table 6, column 1 reports the estimates of the null model. Estimates of independent levels model are in column 2. Parameter estimate of the full model are in column 3. The significance levels of the estimates are indicated by asterisks. Rate parameters that 
describe the amount of change of the two networks ranged from 24.6 to 26.4 in the collaboration network and from 0.33 to 0.65 in the specialty affiliation network. These estimates relate to the number of changes considered by each actor per period in the two networks, and indicates the asymmetric frequency of change in each of these two networks.

Insert Table 6 about here

In general, the results corroborate our expectations. Larger hospitals have more capacity to maintain many specialties and are more active collaborators. In the first two models, larger hospitals are also preferred collaboration partners, especially by other large hospitals, but these results are not sustained in the full model. This means that the effects of organizational "size" is best understood as operating through configurations of network ties. In all three models, a high occupancy rate seems to attract collaboration, but different throughput effects are significant in the three models: whereas throughput ego effect is significant in the null model and the independent level model, throughput similarity is significant in the full model. Distance, LHU, profile and niche are all significant and in expected directions.

Many but not all within-network structural effects are as expected. Reciprocity, transitive closure, outdegree activity, assortativity, and indegree popularity were all significant and in their expected directions. The 4-cycles effects is positive and borderline significant in the full model, indicating a trend towards choices of similar portfolios of internal structures among organizations within the field. Indegree popularity was found to be significant, indicating tendencies towards centralization in receiving activities. All effects need to be interpreted net of other effects included. Indegree activity, for example, was found to be negative, indicating a tendency towards lower outdegrees of those with a high indegree. The model takes into account mechanisms such as indegree popularity 
and outdegree activity that jointly indicate a tendency towards large hospitals with high in- and outdegrees.

The cross-network effects are our central interest here. These effects explain changes in organizational position in the social space created by the interaction of the network of hospitals' collaboration and the network of clinical affiliations. Neither of the cross-network effects explaining the dynamics of specialty affiliation returned significant. We do not find evidence that hospitals that have asymmetric or reciprocal collaborative ties are more likely to assimilate the specialties of their partner. But several cross-network effects explaining collaboration dynamics were found to contribute significantly to the dynamic process. Contrary to our expectations, hospitals with many specialties actually entertain more collaborative ties to other hospitals. This may be because generalist hospitals (with many specialties) serve a triage, gatekeeper or hub function for the system, distributing patients to various smaller niche institutions as required. However, the higher activity of hospitals with more specialties is only present for one-sided relationships. Reciprocal relationships of hospitals with many specialties are less likely than reciprocal relationships of hospitals with fewer specialties. This is revealed by the large negative interaction with the reciprocity effect. Hospitals also collaborate with other hospitals that share the same specialties, but only when the collaboration is reciprocated. This result supports the intuition based on studies of interorganizational relations that have found that similar organizations are more likely to manage the potential rivalry triggered by their similarity by collaborating under conditions of reciprocity (Ring and Van de Ven 1994; Trapido 2007; Uzzi 1996). The main effect of specialty agreement is negative whereas the interaction with reciprocity is significantly positive (and greater in absolute terms). One-sided collaboration seems to be avoided in case of high specialty overlap. 
Next, we compare the multilevel triad census (MLTC) generated by simulations of the three estimated models. Figures $2 \mathrm{a}, 2 \mathrm{~b}$, and $2 \mathrm{c}$ show the centered and scaled distributions of the multilevel motifs that were introduced in figure 1 for the null model, the independent, and the full model, respectively. The red line connects the empirically observed values of the two statistics. The values of all data waves are combined in one plot. A model with a good MLTC fit will show a red line that is close to the means of the distributions. The p-value under each plot indicates the fit, with larger p-values indicating better fit.

Insert Figures 2a, 2b and 2c about here

We can see that the fit of the null model is poor regarding the MLTC and so is the fit of the independent model. For example, the simulations based on the independent model generate too few " 21 " motifs (one-sided collaboration between hospitals that share a specialty) and too many " 20 " motifs (a lack of collaboration between hospitals that share a specialty). The simulation model of the independent levels model in figure $2 \mathrm{~b}$ takes into account the previous waves, a number of covariate related mechanisms (such as size and distance) and all above mentioned structural network effects (such as popularity, assortativity and four-cycles). Predictably, therefore, the fit of the independent model to data is improved over that of the null model in which only covariates were used to model the process.

However, the fit of the full model (Figure 2c) is excellent regarding the MLTC. We can thus not only substantively interpret the estimated parameters but can additionally conclude that the model is well suited to represent the emergence and stability of the empirically observed MLTC. Note that not all but only some of the MLTC motifs are explicitly modeled as effects on either of the two network levels, which contributes to an improved fit. Thus the dynamics of the multilevel 
social space of this organizational field of hospitals is only recreated by employing multilevel relational mechanisms.

\section{Conclusions}

In this paper, we advanced the notion of social space originally proposed by Pattison and Robins (2004) and we extended it to dynamic network systems characterized by the presence of different kind of edges among different kind of nodes. The core feature of these heterogeneous information or mixed mode networks (Kivala et al., 2014) is that the nodes are defined at different levels with relations observable both within and between levels. For this reason, these systems are typically treated as multilevel networks (Wang et al., 2013).

Using data that we collected on a field of health care organizations, we specified and estimated recently derived actor-oriented models for the coevolution of one-mode and two-mode networks (Snijders et al., 2013) to represent concurrent change in the structure of the network linking the organizational nodes and in the internal structure of nodes themselves. In the specific empirical case we have developed, the internal structure of the nodes was defined in terms of changeable portfolios of activities. The structure of the network in which the organizational actors are embedded emerged from dynamic relations of interorganizational collaboration. In the sample we have analyzed for illustrative purposes, nodes are hospital organizations, activities are clinical specialties, and relations of collaboration involve transfer of patients between hospitals - an interorganizational relation that involves intense coordination to support joint problem solving arrangements (Iwashyna, 2012).

The notion of social space we have developed in this paper supported direct analysis of external organizational partner selection decisions, and internal portfolio composition decisions as 
interconnected and mutually dependent. We are not aware of empirical work on organizational communities and fields that has achieved comparable results, despite broad recognition of the analytical challenges posed by the multilevel structure of interorganizational networks (DiMaggio, 1986).

We found strong evidence for endogenous network mechanisms like reciprocity, clustering and degree effects on both network layers (where applicable). Further, node and tie covariates like hospitals' size and distance from one another were important in explaining change in the collaboration and specialty networks. Most importantly though, we found strong evidence for two pairs of cross-network mechanisms. First, hospitals with many specialties (generalist hospitals) were more likely to collaborate with others, however these collaborations were one-sided; hospitals with many specialties were less likely to reciprocate incoming collaboration ties. We note that this result is not obvious, as interorganizational division of labor might make collaboration more likely for smaller, rather than larger hospitals - particularly if size is inversely associated with specialism as it is typically observed to be the case in studies of organizational fields and populations (Carroll, 1985). The inverse association between size and specialism is clearly present in our sample.

Second, hospitals that shared specialties either both collaborated with one another or did not collaborate at all; collaboration in only one direction was unlikely to be observed. We interpreted this as evidence of strategies aiming at reducing the kind of competitive strains introduced by overlapping specialties, and hence similarity in resource requirements (Baum \& Mezias, 1992). Reciprocated collaboration involves transfer of valuable resources, but is also the prerequisite of collective action. Unidirectional collaboration on the other hand tends to be avoided, as unreciprocated transfers of resources are not associated to immediate benefits - at least at the dyadic level. Unlike Stadtfeld et al. (2016), we did not find evidence for the presence of 
assimilation mechanisms that explain how collaborating hospitals might become more similar over time.

Together these mechanisms explained the emergence of the empirically observed MLTC well. We showed this by running computer simulations based on the estimates produced by the empirical models. For each of the MLTC motifs in figure 1, the empirically observed value was very close to the mean of the simulated distribution of values (figure $2 \mathrm{c}$ ). In contrast, two simpler models, one that neglects the role of network structure and one that ignores interdependence between the change processes on both network layers were not able to explain the empirical multilevel triad census (figures $2 \mathrm{a}$ and $2 \mathrm{~b}$ ). Not only we show that the two network layers are interdependent, but we also identify the main dynamic mechanisms underlying such interdependence. We are not aware of empirical work that has revealed with the same clarity the multilevel mechanisms underlying the creation of social spaces.

Our study has several limitations that indicate avenues for future research. The first family of limitations is related to the specific data we have collected. Internal portfolios of activities tend to change slowly over time because economic investments and commitment of resources are rarely perfectly reversible, at least in the short run (Dixit, 2005). As a consequence of this kind of organizational inertia, it could be that longer observation periods are necessary to characterize the dynamics of organizational change more completely. Still, we think that our research design is unique because it allows observation of interconnected processes of internal and external decisions. In other words, we studied the dynamics of interorganizational networks in a social space that is continuously changed by organizations decisions across multiple levels of action. The main cost of this detailed observation scheme is that we had to accept limitations on the length of the sampling period. It is worth adding that, in the specific case of health care, policy interventions and reforms 
tend to generate exogenous changes that would be difficult to incorporate in longitudinal models that focus on endogenous, network-based mechanisms.

The second family of limitations is model-specific, and hence it applies beyond the empirical setting we have examined. The model assumes that processes of change operate only in the short term. This is a problem when investment decisions produce consequences in the more distant future. This is typically the case for capital investment decisions and we cannot rule out that at least some of the change observed in our sample is in fact the consequence of longer-term plans. However, due to the institutional constraints on these organizations we are confident that the majority of the changes observed reflect decisions taken in the context of one budget cycle. Related to this problem, the component elements of social space that are represented in the model (change in network ties and change in internal organizational activities in our specific case) may be characterized by very different "internal" clocks that regulate processes of change happening at different levels (organizational and field levels in our case). The models we have estimated capture these differences in the time structure through the rate parameters, but our models provide no additional indication of how the different "clocks"' that regulate change in the social space may be coupled.

These various limitations advise caution in the empirical extension of the results we have presented. However, we think our paper provides a clear illustration of how to conceptualize multilayered social networks as a social space that is generated and continuously transformed by decisions taken by situated actors. We believe that the approach we have developed also demonstrates the general relevance of multilayered network concepts to specific empirical problems that are central to contemporary research on how organizations adapt to and change their environments. 


\section{References}

Abbott, A., (1997). Of time and space: The contemporary relevance of the Chicago School. Social Forces, 75(4), 1149-1182.

Batagelj, V., \& Bren, M. (1995). Comparing resemblance measures. Journal of Classification, 12(1), 73-90.

Baum, J.A. and Mezias, S.J., (1992). Localized competition and organizational failure in the Manhattan hotel industry, 1898-1990. Administrative Science Quarterly, 580-604.

Bosk, E. A., Veinot, T., and Iwashyna, T. J. (2011). Which patients, and where: A qualitative study of patient transfers from community hospitals. Medical Care, 49(6), 592-598.

Bourdieu, P. (1985). The Social Space and the Genesis of Groups. Theory and Society, 14 (6), $723-$ 44.

Bourdieu, P. (1989). Social Space and Symbolic Power. Sociological Theory, 7 (1), 14-25.

Brunson, J. C. (2015). Triadic analysis of affiliation networks. Network Science, 3(4), 480-508.

Carley, K. (1991). A theory of group stability. American Sociological Review, 56: 331-354.

Carroll, G., (1985). Concentration and specialization: Dynamics of niche width in populations of organizations. American Journal of Sociology, 90(6), 1262-1283.

Cohen, W., \& Levinthal, D. A. (1990). Absorptive capacity: A new perspective on learning and innovation. Administrative Science Quarterly, 35, 128-152.

Cohen, W., \& Levinthal, D. A. (1994). Fortune favors the prepared firm. Management Science, 40, $227-251$. 
Daraganova, G., Pattison, P., Koskinen, J., Mitchell, B., Bill, A., Watts, M. and Baum, S. (2012). Networks and geography: Modelling community network structures as the outcome of both spatial and network processes. Social Networks, 34(1), pp.6-17.

Davis, J.A., \& Leinhardt, S. (1972). The Structure of Positive Interpersonal Relations in Small Groups. In J. Berger (Ed.), Sociological Theories in Progress, Volume 2, 218-251. Boston: Houghton Mifflin.

DiMaggio, P. (1986). Structural analysis of organizational fields: A blockmodeling approach. Research in Organizational Behavior, 8, 335-370.

DiMaggio, P. J., \& Powell, W. W. (1983). The iron cage revisited. Institutional isomorphism and collective rationality in organizational fields. American Sociological Review, 48, 147-60.

Feld, S. L. (1981). The focused organization of social ties. American Journal of Sociology, 86, 1015-1035.

Gittell, J. H. (2002). Coordinating mechanisms in care provider groups: Relational mediator and input uncertainty as a moderator of performance effects, Management Science, 48, 14081426.

Gluckman, M. (1955). The Judicial Process among the Barotse of Northern Rhodesia. Manchester: Manchester University Press.

Greve, H. (2005). Interorganizational learning and heterogeneous social structure. Organization Studies, 26, 1025-1047.

Hains, I. M., Marks, A., Georgiou, A., \& Westbrook, J. I. (2011). Non-emergency patient transport: what are the quality and safety issues? A systematic review. International Journal for Quality in Health Care, 23(1), 68-75. 
Haunschild, P. R., \& Beckman, C. M. (1998). When do interlocks matter? Alternate sources of information and interlock influence. Administrative Science Quarterly, 43, 815-844.

Holland, P. W., \& Leinhardt, S. (1970). A method for detecting structure in sociometric data. American Journal Sociology, 70, 492-513.

Iacobucci, D., \& Wasserman, S. (1990). Social networks with two sets of actors. Psychometrika, $55,707-720$.

Ingram, P., \& Morris, M. W. (2007). Do people mix at mixers? Structure, homophily, and the "Life of the Party". Administrative Science Quarterly, 52, 558-585.

Iwashyna, T. J. (2012). The incomplete infrastructure for interhospital patient transfer. Critical Care Medicine, 40(8), 2470-2478.

Iwashyna, T. J., \& Courey, A. J. (2011). Guided transfer of critically ill patients: where patients are transferred can be an informed choice. Current Opinion in Critical Care, 17, 641-647.

Iwashyna, T. J., Christie, J. D., Moody, J., Kahn, J. M., \& Asch, D. A., (2009). The structure of critical care transfer networks. Medical Care, 47, 787-793.

Kitts, J. A., Lomi, A., Mascia, D., Pallotti, F., \& Quintane, E. (2013). Looking inside interorganizational ties: The dynamics of patient exchange among Italian hospitals, Presented at the American Sociological Association Meeting.

Kivelä, M., Arenas, A., Barthelemy, M., Gleeson, J. P., Moreno, Y., \& Porter, M. A. (2014). Multilayer networks, Journal of Complex Networks, 2(3), 203 - 271.

Kogut, B., Shan, W., \& Walker G. (1992). "The make-or-cooperate decision in the context of an industry network," in Networks and Organisations: structure, form and action, N. Nohria and R. Eccles, eds. Boston: Harvard Business School Press. 
Lazega, E., Jourda, M-T., Mounier, L., \& Stofer, R. (2008). Catching up with big fish in the big pond? Multi-level network analysis through linked design. Social Networks, 30, 159-176.

Lee, B. Y., McGlone, S. M., Song, Y., Avery, T. R., Eubank, S., Chang, C-C., Bailey, R. R., Wagener, D. K., Burke, D. S., Platt, R., \& Huang, S. S. (2011). Social network analysis of patient sharing among hospitals in Orange County, California. American Journal of Public Health, 4, 707-713.

Lomi, A., Lusher, D., Pattison, P., \& Robins, G. (2014a). The focused organization of advice relations: A study in boundary crossing. Organization Science, 25(2), 438-457.

Lomi, A., Mascia, D., Vu, D.Q., Pallotti, F., Conaldi, G., \& Iwashyna, T. J. (2014b). Quality of care and interhospital collaboration: A study of patient transfers in Italy. Medical care, $52,407$.

Lomi, A., \& Pallotti, F. (2012). Relational collaboration among spatial multipoint competitors. Social Networks, 34, 101-111.

Lomi, A., \& Stadtfeld, C. (2014). Social networks and social settings. Developing a coevolutionary view. Kölner Zeitschrift für Soziologie und Sozialpsychologie, 66(1), 395-415.

Lomi, A., Robins, G., \& Tranmer, M. (2016). Introduction to multilevel social networks. Social Networks, 44, 266-268.

Milewicz, K., Hollway, J., Peacock, C., \& Snidal, D. Forthcoming. Beyond Trade: the Expanding Scope of the Non-Trade Agenda in Trade Agreements. Journal of Conflict Resolution.

Milo, R., Shen-Orr, S., Itzkovitz, S., Kashtan, N., Chklovskii, D. \& Alon, U. (2002). Network motifs: Simple building blocks of complex networks. Science, 298, 824-827. 
Mische, A., \& Pattison, P. E. (2000). Composing a civic arena: Publics, projects, and social settings. Poetics, 27, 163-194.

Mische, A., \& White, H. (1998). Between Conversation and Situation: Public Switching Dynamics Across Network Domains. Social Research, 65 (3), 695-724.

Mitsuhashi, H. \& Greve, H., (2009). A matching theory of alliance formation and organizational success: Complementarity and compatibility. Academy of Management Journal, 52(5), 975-995.

Mohr, J. W. (1994). Soldiers, mothers, tramps and others: Discourse roles in the 1907 New York City charity directory. Poetics, 22, 327-357.

Moody, J. (1998). Matrix methods for calculating the triad census. Social Networks, 20, 291-299.

Padgett, J., and Ansell, C. (1993). Robust Action and the Rise of the Medici, 1400-1434. The American Journal of Sociology, 98 (6), 1259-1319.

Padgett, J., and Powell, W. (2012). The Emergence of Organizations and Markets. Princeton University Press.

Pallotti, F., Tubaro, P., \& Lomi, A. (2015). How far do network effects spill over? Evidence from an empirical study of performance differentials in interorganizational networks. European Management Review. (DOI: 10.1111/emre.12052).

Pattison, P. E., \& Robins, G. L. (2002). Neighborhood-based models for social networks. Sociological Methodology, 32, 301-337.

Pattison, P. E., \& Robins, G. L. (2004). Building models for social space: Neighbourhood-based models for social networks and affiliation structures. Mathematics and Social Sciences, 42(168), 11-29. 
Pina-Stranger, A. and Lazega, E., (2011). Bringing personalized ties back in: Their added value for biotech entrepreneurs and venture capitalists interorganizational networks. The Sociological Quarterly, 52(2), pp.268-292.

Powell, W.W., Koput, K.W., \& Smith-Doerr. L. (1996). Interorganizational Collaboration and the Locus of Innovation: Networks of Learning in Biotechnology Administrative Science Quarterly. 41(1):116-145

Reagans, R., \& McEvily, B. (2003). Network structure and knowledge transfer: The effects of cohesion and range. Administrative Science Quarterly, 48, 240-267.

Ripley, R. M., Snijders, T. A. B., Boda, Z., Vörös, A., \& Preciado Lopez, P. (2015). Manual for RSiena. University of Oxford: Department of Statistics; Nuffield College. http://www.stats.ox.ac.uk/ snijders/siena/RSiena_Manual.pdf.

Ring, P. S., \& Van de Ven, A. H. (1994). Developmental processes of cooperative interorganizational relationships. Academy of Management Review, 19, 90-118.

Robinson, V., Goel, V., Macdonald, R. D., \& Manuel, D. (2009). Inter-facility patient transfers in Ontario: Do you know what your local ambulance is being used for?. Health Policy, 4(3), 53-66.

Sethi, D., \& Subramanian, S. (2014). When place and time matter: How to conduct safe interhospital transfer of patients. Saudi Journal of Anaesthesia, 8(1), 104-113.

Shipilov, A. V. and Li, S. X. (2010) 'The Missing Link: The Effect of Customers on the Formation of Relationships among Producers in the Multiplex Triads', Organization Science 23(2): 472-91. 
Snijders, T. A. B. (2016). The multiple flavours of multilevel issues for networks. In E. Lazega and T. A. B. Snijders (Eds), Multi-level network analysis for the social sciences: Theory, methods and applications (pp. 15-46). Springer.

Snijders, T. A. B., \& Stokman, F. N. (1987). Extensions of triad counts to networks with different subsets of points and testing underlying random graph distributions. Social Networks, 9, 249-275.

Snijders, T. A. B., Lomi, A., \& Torlo', V. J. (2013). A model for the multiplex dynamics of twomode and one-mode networks, with an application to employment preference, friendship, and advice. Social Networks, 35, 265-276.

Snijders, T. A.B., van de Bunt, G. G., \& Steglich, C. E.G. (2010). Introduction to stochastic actorbased models for network dynamics. Social Networks, 32, 44-60.

Sohn, M-W. (2001). Distance and cosine measures of niche overlap. Social Networks, 23, 141165.

Stadtfeld, C., Mascia, D., Pallotti, F., \& Lomi, A. (2016). Assimilation and differentiation: A multilevel perspective on organizational and network change. Social Networks, 44, 363 374.

Tortoriello, M., \& Krackhardt, D. (2010). Activating cross-boundary knowledge: The role of Simmelian ties in the generation of innovations. Academy of Management Journal, 53(1), 167-181.

Tortoriello, M., Reagans, R., \& McEvily, W. (2012). Bridging the knowledge gap: The influence of strong ties, network cohesion, and network range on the transfer of knowledge between organizational units. Organization Science, 23, 1024-1039. 
Toth, F. (2014). Health care regionalization in Italy. Working Paper presented at the 23rd World Congress of Political Science, Montréal, July 19-14.

Trapido, D. (2007). Competitive embeddedness and the emergence of interfirm cooperation. Social Forces, 86(1), 165-191.

Uzzi, B. (1996). The sources and consequences of embeddedness for the economic performance of organizations: the network effect. American Sociological Review, 61, 674-698.

Veinot, T. C., Bosk, E. A., Unnikrishnan, K. P., \& Iwashyna, T. J. (2012). Revenue, relationships and routines: The social organization of acute myocardial infarction patient transfers in the United States. Social Science \& Medicine, 75, 1800-1810.

Verbrugge, L. M. (1979). Multiplexity in Adult Friendships. Social Forces, 57 (4), 1286-1309.

Wagner, J., Iwashyna, T. J. \& Kahn, J. M. (2013). Reasons underlying interhospital transfers to an academic medical intensive care unit. Journal of Critical care, 28(2), 202-208.

Wang, P., Robins, G., Pattison, P., \& Lazega, E. (2013). Exponential random graph models for multilevel networks. Social Networks, 35, 96-115.

Wasserman, S. (1977). Random directed graph distributions and the triad census in social networks. Journal of Mathematical Sociology, 5, 61-86.

Wasserman, S., \& Faust, K. (1994). Social Network Analysis: Methods and Applications. Cambridge: Cambridge University Press.

Wasserman, S., \& Iacobucci, D. (1991). Statistical modelling of one-mode and two-mode networks: Simultaneous analysis of graphs and bipartite graphs. British Journal of Mathematical and Statistical Psychology, 44, 13-43. 

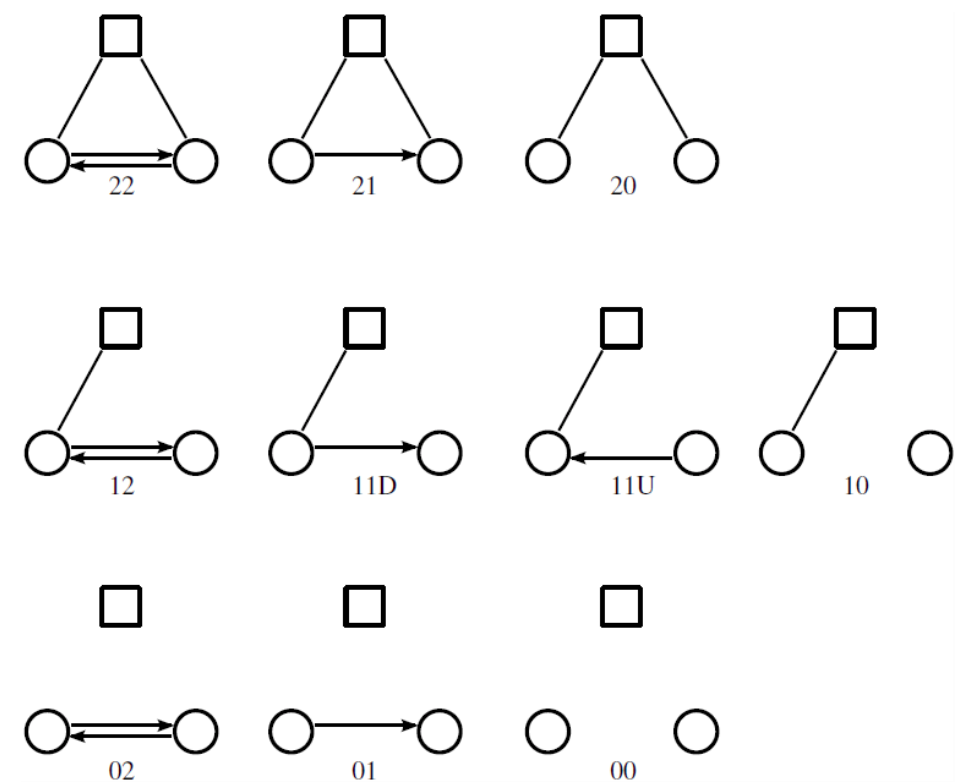

Figure 1: Triadic motifs in the multilevel triad census

Table 1: Descriptives of the dichotomized collaboration networks

\begin{tabular}{|c|c|c|c|c|c|}
\hline & \multicolumn{5}{|l|}{ Wave } \\
\hline & 1 & 2 & 3 & 4 & 5 \\
\hline$N$ hospitals & 110 & 110 & 107 & 103 & 100 \\
\hline Density & 0.186 & 0.183 & 0.186 & 0.193 & 0.197 \\
\hline \multirow[t]{2}{*}{ Avg. degree } & 20.273 & 19.94 & 20.23 & 21.03 & 21.52 \\
\hline & & 5 & 0 & 0 & 5 \\
\hline$N$ ties & 2230 & 2194 & 2105 & 2027 & 1955 \\
\hline
\end{tabular}


Table 2: Collaboration network: Change Statistics

\begin{tabular}{|c|c|c|c|c|}
\hline & Period & & & \\
\hline & $1-2$ & $2-3$ & $3-4$ & $4-5$ \\
\hline $0 \rightarrow 0$ & 9111 & 8578 & 7898 & 7351 \\
\hline $0 \rightarrow 1$ & 649 & 605 & 587 & 559 \\
\hline $1 \rightarrow 0$ & 685 & 659 & 581 & 594 \\
\hline $1 \rightarrow 1$ & 1545 & 1500 & 1440 & 1396 \\
\hline Distance & 1334 & 1264 & 1168 & 1153 \\
\hline Jaccard C. & 0.537 & 0.543 & 0.552 & 0.548 \\
\hline
\end{tabular}

Table 3: Specialty network: Change statistics

\begin{tabular}{|c|c|c|c|c|}
\hline & Period & & & \\
\hline & $1-2$ & $2-3$ & $3-4$ & $4-5$ \\
\hline $0 \rightarrow 0$ & 5208 & 5264 & 5261 & 5291 \\
\hline $0 \rightarrow 1$ & 7 & 17 & 29 & 26 \\
\hline $1 \rightarrow 0$ & 73 & 26 & 56 & 44 \\
\hline $1 \rightarrow 1$ & 982 & 963 & 924 & 909 \\
\hline Distance & 34 & 33 & 55 & 59 \\
\hline Jaccard C. & 0.925 & 0.957 & 0.916 & 0.928 \\
\hline
\end{tabular}


Table 4: Exogenous covariates

\begin{tabular}{lcccr}
\hline Variable name & Mean & SD & Min & Max \\
\hline Distance & 4.990 & 4.030 & 0 & 22.260 \\
Niche overlap & 0.112 & 0.172 & 0 & 0.811 \\
Same LHU & 0.100 & 0.301 & 0 & 1 \\
Same profile & 0.339 & 0.473 & 0 & 1 \\
Size/100 & 1.827 & 2.362 & 0 & 16.850 \\
Occupancy & 0.686 & 0.211 & 0.027 & 1.525 \\
Throughput & 8.207 & 1.343 & 0 & 11.075 \\
\hline
\end{tabular}


Table 5: Network effects: Definitions and description

\begin{tabular}{|c|c|c|}
\hline Mechanism & Diagram & Equation \\
\hline $\begin{array}{l}\text { COLLABORATION } \\
\text { Specialty Activity }\end{array}$ & & $s_{21 i}^{X}(x, y)=\sum x_{i j}\left(\sqrt{y_{i+}}-\sqrt{y}\right)$ \\
\hline $\begin{array}{l}\text { Specialty Activity } \\
\text { Reciprocity }\end{array}$ & & $s_{22 i}^{X}(x, y)=\sum_{j} x_{i j} x_{j i}\left(\sqrt{y_{i+}}-\sqrt{y}\right)$ \\
\hline Specialty Popularity & & $s_{23 i}^{X}(x, y)=\sum_{j} x_{i j}\left(\sqrt{y_{j+}}-\sqrt{y}\right)$ \\
\hline Specialty Agreement & & $s_{24 i}^{X}(x, y)=\sum_{j, h} x_{i j} y_{i h} y_{j h}$ \\
\hline $\begin{array}{l}\text { Specialty Agreement } \\
\text { Reciprocity }\end{array}$ & & $s_{25 i}^{X}(x, y)=\sum_{j, h} x_{i j} x_{j i} y_{i h} y_{j h}$ \\
\hline $\begin{array}{l}\text { SPECIALTY } \\
\text { Collaboration } \\
\text { Agreement }\end{array}$ & & $s_{30 i}^{Y}(x, y)=\sum_{j, h} x_{i j} y_{i h} y_{j h}$ \\
\hline $\begin{array}{l}\text { Collaboration } \\
\text { Agreement } \\
\text { Reciprocity }\end{array}$ & & $s_{31 i}^{Y}(x, y)=\sum_{j, h} x_{i j} x_{j i} y_{i h} y_{j h}$ \\
\hline
\end{tabular}


Table 6: Model estimates (standard errors in parentheses)

\begin{tabular}{|c|c|c|c|c|c|c|}
\hline \multirow[b]{2}{*}{ Collaboration Network } & \multicolumn{2}{|c|}{ Null Model } & \multicolumn{2}{|c|}{ Independent } & \multicolumn{2}{|c|}{ Full Model } \\
\hline & Est. & s.e. & Est. & s.e. & Est. & s.e. \\
\hline Outdegree & $-1.33 * * *$ & $(0.02)$ & $-3.61 * * *$ & $(0.09)$ & $-3.36 * * *$ & $(0.11)$ \\
\hline Reciprocity & $0.44 * * *$ & $(0.03)$ & $1.17 * * *$ & $(0.20)$ & 0.32 & $(0.21)$ \\
\hline Distance & $-0.10 * * *$ & $(0.00)$ & $-0.04 * * *$ & $(0.00)$ & $-0.06 * * *$ & $(0.01)$ \\
\hline Niche overlap & $-0.53 * * *$ & $(0.09)$ & $-0.24 * *$ & $(0.08)$ & $-0.27 * *$ & $(0.09)$ \\
\hline Same LHU & $0.52 * * *$ & $(0.04)$ & $0.80 * * *$ & $(0.04)$ & $0.78 * * *$ & $(0.04)$ \\
\hline Same profile & $0.11 * * *$ & $(0.03)$ & $0.08 * *$ & $(0.03)$ & $0.08 * *$ & $(0.03)$ \\
\hline Size alter & $0.25^{* * *}$ & $(0.01)$ & $0.05 * * *$ & $(0.01)$ & 0.02 & $(0.02)$ \\
\hline Size ego & $0.12 * * *$ & $(0.02)$ & $0.06 * * *$ & $(0.02)$ & $0.07 * * *$ & $(0.02)$ \\
\hline Size similarity & $2.70 * * *$ & $(0.24)$ & $0.46^{*}$ & $(0.22)$ & -0.10 & $(0.24)$ \\
\hline Occupancy alter & $1.05 * * *$ & $(0.07)$ & $0.23 * *$ & $(0.08)$ & $0.27 * * *$ & $(0.08)$ \\
\hline Occupancy ego & $0.60 * * *$ & $(0.08)$ & $0.29 * *$ & $(0.09)$ & $0.26 * *$ & $(0.09)$ \\
\hline Occupancy similarity & 0.22 & $(0.12)$ & 0.02 & $(0.12)$ & 0.06 & $(0.12)$ \\
\hline Throughput alter & 0.02 & $(0.02)$ & -0.03 & $(0.02)$ & -0.03 & $(0.03)$ \\
\hline Throughput ego & $0.31 * * *$ & $(0.02)$ & $0.07 * * *$ & $(0.02)$ & 0.01 & $(0.03)$ \\
\hline Throughput similarity & $-0.69 * *$ & $(0.21)$ & -0.10 & $(0.21)$ & $0.51 *$ & $(0.26)$ \\
\hline Transitive closure & & & $0.51 * * *$ & $(0.07)$ & $0.45^{* * *}$ & $(0.07)$ \\
\hline Cyclic closure & & & 0.02 & $(0.06)$ & 0.06 & $(0.06)$ \\
\hline Indegree-popularity & & & $0.02 * * *$ & $(0.002)$ & $0.02 * * *$ & $(0.002)$ \\
\hline Outdegree-activity & & & $0.01 * * *$ & $(0.002)$ & $0.01 * * *$ & $(0.002)$ \\
\hline Indegree-activity & & & $-0.01 * * *$ & $(0.003)$ & $-0.02 * * *$ & $(0.003)$ \\
\hline Out-in degree assortativity & & & $0.03 * * *$ & $(0.004)$ & $0.03 * * *$ & $(0.004)$ \\
\hline
\end{tabular}


Transitive closure $\mathrm{x}$

$$
-0.37 * * * \quad(0.11) \quad-0.09
$$

Reciprocity

Specialty activity

$0.28 * * * \quad(0.04)$

Specialty activity

$-0.59 * * * \quad(0.08)$

reciprocity

0.01

(0.03)

Specialty popularity

$-0.02 * \quad(0.01)$

Specialty agreement

$0.08 * * * \quad(0.02)$

Specialty agreement reciprocity

\begin{tabular}{|c|c|c|c|c|c|c|}
\hline Affiliation Network & & & & & & \\
\hline Outdegree & $-1.24 * * *$ & $(0.10)$ & $-2.34 * * *$ & $(0.17)$ & $-2.44 * * *$ & $(0.17)$ \\
\hline Size ego & $0.32 * * *$ & $(0.08)$ & $0.23 * * *$ & $(0.06)$ & $0.23 * * *$ & $(0.06)$ \\
\hline 4-cycles & & & $0.01 * * *$ & $(0.001)$ & 0.003 & $(0.002)$ \\
\hline Indegree-popularity & & & $0.01 *$ & $(0.01)$ & 0.01 & $(0.01)$ \\
\hline Collaboration agreement & & & & & 0.05 & $(0.05)$ \\
\hline $\begin{array}{l}\text { Collaboration agreement } \\
\text { reciprocity }\end{array}$ & & & & & 0.02 & $(0.05)$ \\
\hline Iterations & 8055 & & & & 8783 & \\
\hline
\end{tabular}

$* * * \mathrm{p}<0.001 ; * * \mathrm{p}<0.01 ; * \mathrm{p}<0.05 ; \mathrm{p}<0.1$ 


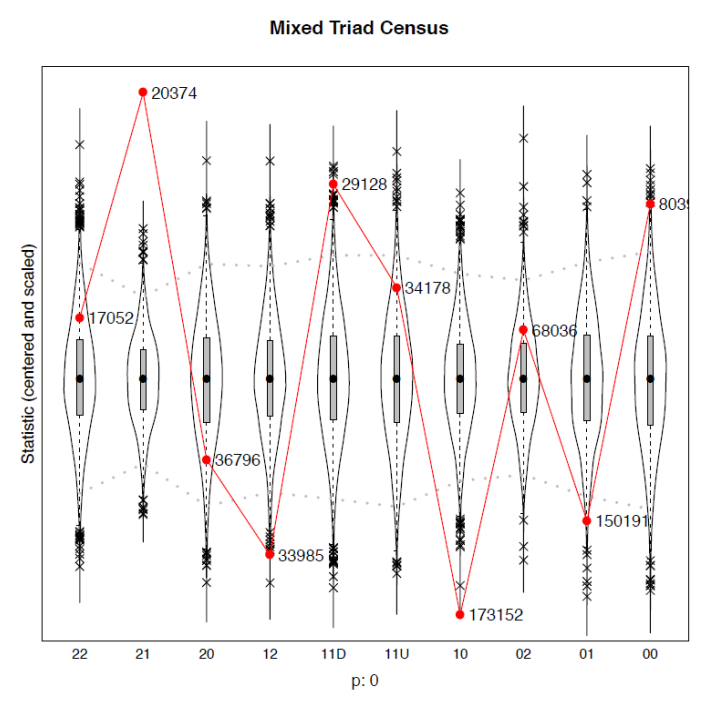

(a)
Mixed Triad Census

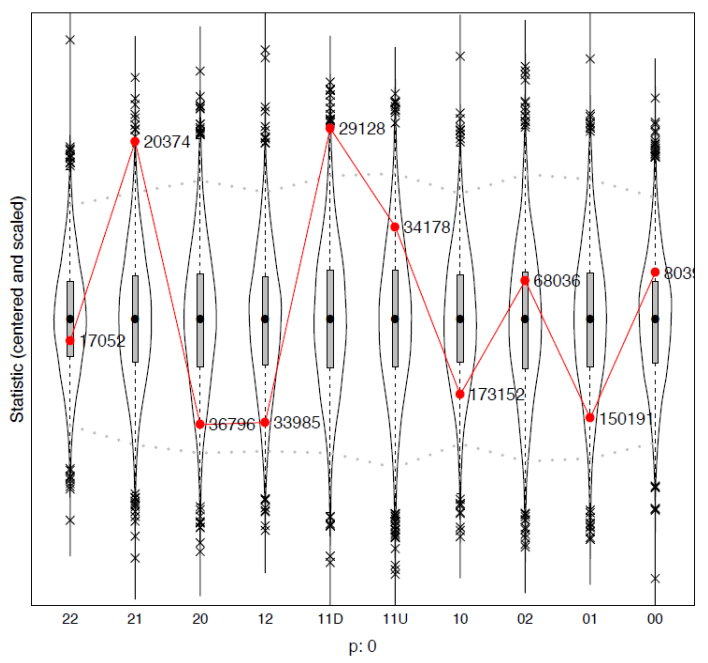

(b)

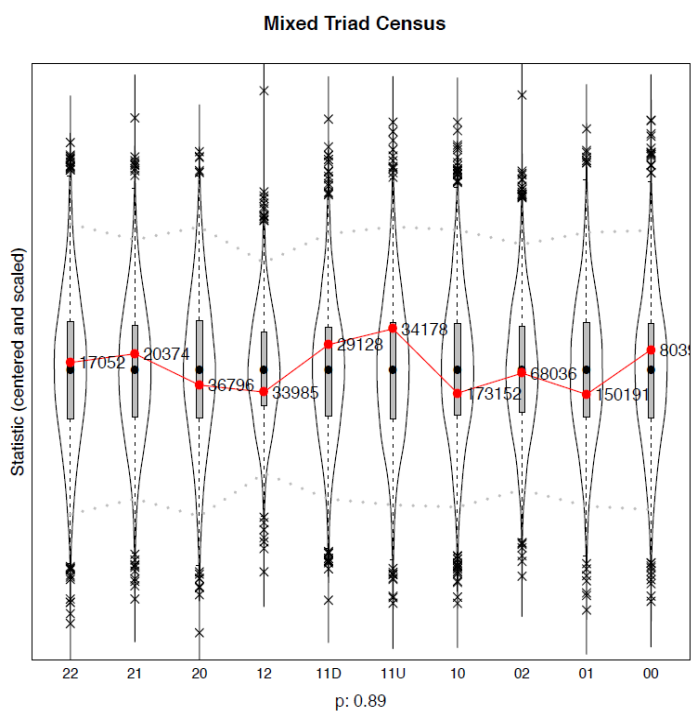

(c)

Figure 2a, b, and c: Goodness of fit diagnostic violin plots of null model (estimates in Table 6, column 1, independent model (estimates in Table 6, column 2 and full model (estimates in Table 6, column 3) 